CARDIOVASCULAR MEDICINE

\title{
Predicting sudden death in patients with mild to moderate chronic heart failure
}

\author{
M T Kearney, K A A Fox, A J Lee, W P Brooksby, A M Shah, A Flapan, R J Prescott, R Andrews, \\ P D Batin, D L Eckberg, N Gall, A G Zaman, H S Lindsay, J Nolan
}

Heart 2004;90:1137-1143. doi: 10.1136/hrt.2003.021733

See end of article for authors' affiliations

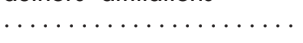

Correspondence to: DrM Kearney, Department of Cardiology, Kings College, Bessemer Road, London SE5 9PJ, UK; mark.kearney@kcl.ac.uk

Accepted

13 November 2003

\begin{abstract}
Objectives: To explore the relation between non-invasive measures of cardiac function and sudden cardiac death, as well as the development and utility of an index integrating these variables to identify patients at increased risk of this mode of death.

Design: UK-HEART (United Kingdom-heart failure evaluation and assessment of risk trial) was a prospective study conducted between December 1993 and April 2000. The study was specifically designed to identify non-invasive markers of death and mode of death among patients with chronic heart failure.

Setting: 8 UK general hospitals.

Main outcome measures: Death and mode of death.

Results: 553 patients aged a mean (SD) of $63(10)$ years, in New York Heart Association functional class 2.3 (0.02), recruited prospectively. After 2365 patient-years' follow up, 201 patients had died $(67$ suddenly). Predictors of sudden death were greater cardiothoracic ratio, QRS dispersion, QT dispersion corrected for rate (QTc) across leads V1-V6 on the 12 lead ECG, and the presence of non-sustained ventricular tachycardia. The hazard ratio and $95 \%$ confidence intervals $(\mathrm{Cl})$ of sudden death for a $10 \%$ increase in cardiothoracic ratio was $1.43(95 \% \mathrm{Cl} 1.20$ to 1.71$)$, for a $10 \%$ increase in QRS dispersion $1.11(95 \% \mathrm{Cl} 1.04$ to 1.19$)$, for the presence of non-sustained ventricular tachycardia 2.03 (95\% Cl 1.27 to 3.25), and for a 10\% increase in QTc dispersion across leads $\mathrm{V} 1-\mathrm{V} 61.03$ (95\% Cl 1.00 to 1.07 ) (all $\mathrm{p}<0.04)$. An index derived from these four factors performed well in identifying patients specifically at increased risk of sudden death.

Conclusions: Results show that an index derived from three widely available non-invasive investigations has the potential to identify ambulant patients with chronic heart failure at increased risk of sudden death. This predictive tool could be used to target more sophisticated investigations or interventions aimed at preventing sudden death.
\end{abstract}

C hronic heart failure (CHF) is the most prevalent cardiovascular disorder in western societies. ${ }^{1}$ Despite the use of new drugs, patients with relatively mild symptoms of CHF have a mortality approaching $10 \%$ a year. $^{2}$ At least half of these deaths occur suddenly without warning. In the Framingham study, the sudden death rate for patients with CHF was almost 10 times the general age adjusted population rate. ${ }^{3}$ The majority of patients with $\mathrm{CHF}$ have relatively mild to moderate symptoms but are still at significant risk of sudden death compared with patients with more substantial functional impairment. ${ }^{245}$ Treatments aimed at preventing sudden death and non-sudden death require different strategies. Over the past 10 years implantable cardioverter-defibrillators have emerged as a potentially effective treatment to prevent sudden death. ${ }^{67}$ However, trials have focussed on patients with evidence of significantly impaired left ventricular function and the population studied has not been selected on the basis of symptoms and signs of CHF.

Over $50 \%$ of patients with myocardial infarction who later die suddenly have well preserved left ventricular function. ${ }^{8}$ Within the large population of patients with relatively preserved left ventricular function and mild to moderate CHF there is a subgroup at particularly high risk of sudden death. ${ }^{9}$ Establishing reliable non-invasive predictors of sudden death is hence important. There is no accurate method of risk stratifying ambulant patients with the CHF phenotype who are at increased risk of this mode of death. The UK-HEART (United Kingdom-heart failure evaluation and assessment of risk trial) prospectively explored the prognostic utility of non-invasive measures of cardiac autonomic, electrical, and mechanical function along with plasma electrolytes and renal function in ambulant outpatients with CHF. The present report describes the relation between these variables and sudden cardiac death and the development and utility of an index integrating these variables to identify patients at increased risk of this mode of death.

\section{METHODS}

\section{Study population}

UK-HEART was a multicentre study carried out in eight UK institutions. We have previously published details of the study design. ${ }^{10-12}$ In brief, ambulant outpatients of either sex 18 to 85 years old were recruited. Patients were eligible for the study if they had stable clinical signs and symptoms of CHF present for at least three months. Patients were in New York Heart Association (NYHA) functional class I-III and had objective evidence of cardiac dysfunction (pulmonary venous congestion, pulmonary oedema or a cardiothoracic ratio $>0.55$ on at least one chest radiograph, or a documented radionuclide or echocardiographic ejection fraction of

Abbreviations: $\mathrm{CHF}$, chronic heart failure; $\mathrm{Cl}$, confidence interval; MADIT II, multicentre automatic defibrillator implantation trial II; NYHA, New York Heart Association; ROC, receiver operating characteristic; QTc, QT interval corrected for rate; UK-HEART, United Kingdom-heart failure evaluation and assessment of risk trial 
$<45 \%$ ). A number of carefully performed population studies have reported that up to $40 \%$ of incident and $50-60 \%$ of prevalent CHF cases occur in the setting of preserved left ventricular function. ${ }^{13}$ Consonant with this, a reduced ejection fraction was not an absolute requirement for entry into UK-HEART. Patients were excluded if they had a comorbid condition associated with impaired autonomic function (including diabetes mellitus). Other exclusion criteria have been documented previously..$^{10-12}$ Studies were carried out in accordance with the standards of the local ethical committees and with the Declaration of Helsinki. All patients gave written informed consent to take part in the study.

\section{Clinical data collection}

At the time of recruitment, a case record form detailing baseline clinical and demographic data was completed for all patients. An erect posteroanterior chest radiograph was obtained and the cardiothoracic ratio was measured. A venous blood sample was taken at rest for assessment of electrolyte concentration and of renal and liver function. Two dimensional and $M$ mode echocardiography was performed in accordance with the American Society of Echocardiography recommendations. Left ventricular cavity dimensions and ejection fraction were derived from the $\mathrm{M}$ mode echocardiograms with standard formulas. Study patients were registered with the UK national death reporting scheme, which notified the steering committee of all deaths.

\section{Twelve lead ECG analysis}

Standard 12 lead ECGs were recorded at $25 \mathrm{~mm} / \mathrm{s}$ and analysed by a senior cardiologist blinded to patient characteristics. The QT interval and QRS durations were measured manually and in the case of QT, corrected for rate (QTc) as previously described. ${ }^{11}$ To evaluate the possible role of local dispersion of repolarisation in predicting sudden death we calculated QTc dispersion across leads Vl-V6. ${ }^{14}$ QTC and QRS dispersions were defined as the difference between the maximum and minimum QTc or QRS values, respectively, occurring in any of the 12 ECG leads or leads V1-V6. Left ventricular hypertrophy was assessed by the Sokolow-Lyon voltage criteria. ${ }^{15}$

\section{Ambulatory ECG}

Twenty four hour ambulatory ECGs (Tracker, Reynolds Medical, Hertford, UK) were obtained from all patients during normal, unrestricted, out of hospital activity. Recordings were analysed with a Reynolds Medical Pathfinder system by independent technical staff blinded to patient characteristics. Time and frequency domain were analysed as previously described ${ }^{10}{ }^{12}{ }^{16}$ Non-sustained ventricular tachycardia was defined as three or more consecutive ventricular extrasystoles at a rate $>120$ beats $/ \mathrm{min}$.

\section{Classification of mode of death}

Classification criteria for the cause of death were defined before the study commenced and were based on established definitions. ${ }^{17}$ All deaths reported to the steering committee were evaluated by at least two senior physicians, who reviewed death certificates, necropsy findings, and hospital and general practitioners' records. The mode of death was classified as follows: (1) sudden if it occurred within one hour of a change in symptoms or during sleep or while the patient was unobserved and had previously been clinically stable; (2) progressive heart failure if death occurred after a documented period of symptomatic or haemodynamic deterioration; (3) other cardiovascular if death did not occur suddenly and was not associated with progression of heart failure-this category included patients who died of acute coronary syndromes; and (4) non-cardiovascular death.

\section{Statistical analysis}

The analytical goal was to identify independent predictors of sudden cardiac death. Additionally, we aimed to construct a simple predictive score that could be used in routine clinical practice to identify patients with CHF who are at increased risk of sudden death. Statistical analyses were completed with SPSS (version 10; SPSS Inc, Chicago, Illinois, USA), SAS (versions 6.12 and 8.2; SAS Institute Inc, Cary, North Carolina, USA), and S-Plus (version 2000 Professional Release 1, MathSoft Inc, Cambridge, Massachusetts, USA). Descriptive group data are given as mean (SD) for continuous variables (medians and quartiles for non-normally distributed data) and percentages for categorical data.

\section{Cox proportional hazards regression}

Stepwise Cox proportional hazards regression ${ }^{18}$ was used to determine which measurements were significantly related to mortality during the follow up period. To maximise the number of observations available for the present analysis, missing values for any variable were estimated by multiple regression from their relation with other variables by using the missing data facility within SPSS. Natural logarithms were used for all heart rate variability measurements and cardiothoracic ratio.

Variables entered into the sudden death model

In initial univariate and multivariate analyses neither time nor frequency domain measurements of heart rate variability were significant independent predictors of sudden death and therefore were not considered in the present model (table 1).

Age, sex, the presence of non-sustained ventricular tachycardia and left ventricular hypertrophy, left ventricular end diastolic and end systolic diameters, ejection fraction, sodium, potassium, urea, and creatinine concentrations, the logarithm of the cardiothoracic ratio, natural logarithms of QTc dispersion, QTc dispersion across leads V1-V6, maximum QTC interval, and QRS dispersion were all univariate predictors of sudden death and were therefore entered into the statistical model. The assumption of proportional hazards was tested and was fulfilled. Hazard ratios and 95\% confidence intervals (CI) are presented. Kaplan-Meier cumulative mortality curves were produced to display the proportion of patients free from sudden death over time for each of the independent predictors.

\section{Derivation and discrimination of predictive model}

A prognostic index to predict sudden death was derived for each patient based on the Cox proportional hazards model. ${ }^{19}$ To maximise the information supplied from the Cox model, the parameter estimates were used to obtain an index. A score for each of the significant independent predictors was calculated for each patient by multiplying their value of the factor with the parameter estimate. A sudden cardiac death index for each patient was then derived from the sum of these scores. We used receiver operating characteristic (ROC) analysis to quantify the diagnostic accuracy of our index. ROC curves plot the sensitivity against 1 - specificity by varying the threshold value for the test. They illustrate which threshold is a good compromise between high sensitivity and high specificity. ${ }^{20}$ The area under the curve (C statistic) was calculated. An area of 0.5 indicates that the test results are no better than those obtained by chance, whereas an area of 1.0 indicates a perfectly sensitive and specific test.

\section{RESULTS}

\section{Characteristics of UK-HEART population}

Five hundred and fifty three patients were recruited. The patients' mean (SEM) age was 62.7 (0.41) years (range 18-85 years) and $76 \%$ were men. Two per cent were in NYHA class I, 
Table 1 Univariate logistic regression for time and frequency domain heart rate variability measurements and sudden death status at five years

\begin{tabular}{lll}
\hline Measurement & $\begin{array}{l}\text { Odds ratio for sudden death for every } \\
10 \% \text { decrease in variable }(95 \% \mathrm{Cl})\end{array}$ & p Value \\
\hline SDNN (ms) & $0.95(0.88$ to 1.02$)$ & 0.15 \\
Very low frequency power $\left(\mathrm{ms}^{2}\right)$ & $1.01(0.95$ to 1.08$)$ & 0.63 \\
Low frequency power $\left(\mathrm{ms}^{2}\right)$ & $0.96(0.78$ to 1.19$)$ & 0.74 \\
High frequency power $\left(\mathrm{ms}^{2}\right)$ & $0.98(0.84$ to 1.14$)$ & 0.84 \\
Total power $\left(\mathrm{ms}^{2}\right)$ & $1.00(0.95$ to 1.05$)$ & 0.80 \\
\hline Cl, confidence interval; SDNN, standard deviation of all NN intervals. &
\end{tabular}

$59 \%$ in class II, and 39\% in class III. The average NYHA functional class was $2.3(0.02)$. Mean (SD) creatinine concentration was 121 (1.8) $\mu \mathrm{mol} / \mathrm{l}$ (range $60-340 \mu \mathrm{mol} / \mathrm{l}$ ). Mean (SD) sodium concentration was $140(0.14) \mathrm{mmol} / \mathrm{l}$ (range 122-148 mmol/l). Seventy six per cent of patients had ischaemic heart disease as the cause of their heart failure, 199 (36\%) had non-sustained ventricular tachycardia, and 54 $(9.8 \%)$ had left ventricular hypertrophy. The majority of patients $(81 \%)$ were treated with angiotensin converting enzyme inhibitors (mean (SD) dose of 12.4 (0.34) mg (enalapril equivalent)) and loop diuretics (97\% of patients, mean (SD) furosemide (frusemide) dose 75.4 (2.9) $\mathrm{mg}$ ). Nineteen per cent of patients were taking digoxin (mean dose $198(7.12) \mu \mathrm{g}), 14 \%$ amiodarone (all patients $200 \mathrm{mg}$ ), and $7.9 \%$ atenolol (mean dose 43.7 (1.6) $\mathrm{mg}$ ). Data on five year survival status was available for all patients. At five years (2365 patient-years), 201 patients had died (mean annual mortality rate $7.3 \%$ ) with 67 sudden deaths.

\section{Predictors of sudden cardiac death}

Cox multivariate independent predictors of sudden death shown in table 2 were cardiothoracic ratio, QRS dispersion, QTc dispersion across leads Vl-V6, and the presence of nonsustained ventricular tachycardia. Kaplan-Meier curves (fig 1) show the proportion of patients free from sudden death with variables dichotomised by median values or in the case of non-sustained ventricular tachycardia its presence or absence.

In a preliminary report ${ }^{10}$ (follow up 482 (161) days) with only 18 sudden deaths serum potassium was a weak predictor of sudden death. In the present analysis, which has far greater statistical power and incorporated QTc and QRS measurements in the sudden death model, potassium is not an independent predictor of sudden death.

\section{QRS dispersion or maximum QRS as a predictor of sudden death}

The recently published MADIT II (multicentre automatic defibrillator implantation trial II $)^{7}$ suggested that patients with previous myocardial infarction and an ejection fraction $<30 \%$ with a QRS duration > 120 ms benefited most from implantable cardioverter-defibrillators. To explore the possibility that the prognostic value of QRS dispersion was simply a reflection of maximum QRS duration, we analysed the same model but with QRS dispersion being replaced by QRS minimum and QRS maximum durations. While QRS maximum alone also gave predictive information it did not reach the same level of significance as QRS dispersion.

\section{Characteristics of survivors and of patients who died suddenly and non-suddenly}

Comparing the characteristics of survivors, patients who died suddenly, and those who died non-suddenly showed a significant difference between the three groups. However, the value of any measurement to predict sudden death is in its ability to identify patients at increased risk of sudden death and to discriminate between patients who die suddenly and those who die non-suddenly. In the present cohort the phenotypes of these patients were very similar in demographic, echocardiographic, and ECG data. No single measurement alone separated the two groups (table 3). Hence we integrated the four independent predictors of sudden death to develop a predictive model.

\section{Development of predictive model of sudden death}

To explore the utility of combining the four independent predictors to identify patients at increased risk of sudden death, we derived a prognostic index based on the importance of each predictor in the Cox model. The natural logarithm of the patient's cardiothoracic ratio, QRS dispersion, and QTc dispersion was calculated. These figures were then multiplied by $3.75,1.10$, and 0.36 , respectively, and the sum was calculated. An additional score of 0.71 was added if non-sustained ventricular tachycardia was present (no score was added if it was not present). The resulting score ranged from approximately 1.28 to 3.80 with a higher score denoting increased risk of sudden cardiac death within five years. When dividing the UK-HEART population into deciles, for patients with the highest score (56 patients) the index had a positive predictive value of 37.5 , negative predictive value of 90.7, sensitivity of 31.3 , and specificity of 92.8 . We used the bootstrap method (S-Plus) to validate the model and found that with 20000 bootstrap samples, $95 \%$ of sensitivity values fell between $53.5 \%$ and $76.6 \%$ indicating that the index has good potential to be useful in other similar populations of CHF patients.

Table 2 Cox Multivariate predictors of sudden death at five years

\begin{tabular}{|c|c|c|c|c|}
\hline Variable & $\begin{array}{l}\text { Mean (SEM) } \\
\text { parameter } \\
\text { estimates }\end{array}$ & $\chi^{2}$ & $\begin{array}{l}\text { Cox multivariate } \\
\text { analysis hazard ratio } \\
(95 \% \mathrm{Cl})\end{array}$ & $p$ Value \\
\hline Cardiothoracic ratio (10\% increase) & $3.75(0.96)$ & 15.3 & $1.43(1.20$ to 1.71$)$ & 0.001 \\
\hline NSVT (present) & $0.71(0.24)$ & 8.7 & 2.03 (1.27 to 3.25 ) & 0.003 \\
\hline QRS dispersion ( $10 \%$ increase) & $1.10(0.36)$ & 9.2 & 1.11 (1.04 to 1.19 ) & 0.002 \\
\hline QTc dispersion V1-V6 (10\% increase) & $0.36(0.17)$ & 4.4 & $1.03(1.00$ to 1.07$)$ & 0.035 \\
\hline
\end{tabular}



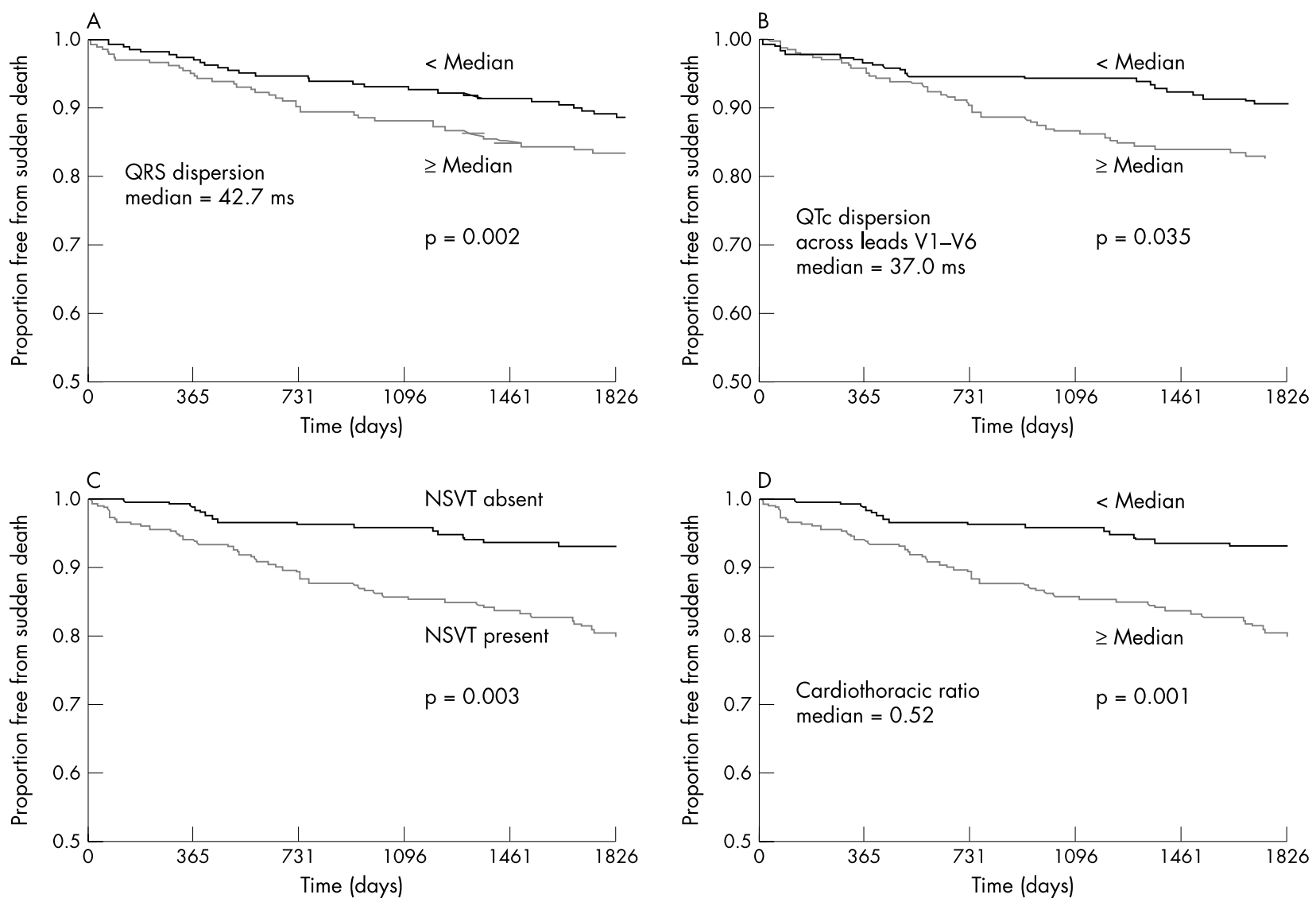

Figure 1 Kaplan-Meier curves showing proportions of patients free from sudden death over five years when patients are dichotomised by median value of (A) QRS dispersion, (B) QT dispersion across leads V1-V6 corrected for rate (QTc), (C) the presence or absence of non-sustained ventricular tachycardia (NSVT), and (D) cardiothoracic ratio dichotomised by median.

Accuracy of the predictive model in CHF subgroups

To further explore the diagnostic accuracy of this index we constructed ROC curves (not shown). The C statistic for this index to predict sudden death within the whole UK-HEART population was 0.71 (95\% CI 0.64 to 0.78 ). We further analysed patients with ischaemic heart disease and ejection fractions less than $45 \%$. Applied to this patient group $(\mathrm{n}=262)$ the index improved in its predictive value and discrimination (C statistic $0.76,95 \%$ CI 0.68 to 0.84 ). When the group of patients with ejection fractions $<45 \%$ and ischaemic heart disease was divided into deciles, for patients with the highest score ( 39 patients) the index had a positive predictive value of 38.5, negative predictive value of 89.7, sensitivity of 39.5, and specificity of 89.3. We used the bootstrap method to validate this model and found that with 20000 bootstrap samples, 95\% of sensitivity values fell between $54.7 \%$ and $84.2 \%$ indicating that the index has good potential to be useful in another similar population of CHF patients.

\section{DISCUSSION}

It is well established that patients with mild to moderate CHF are at relatively greater risk of sudden death than patients with more substantial functional impairment. ${ }^{5}$ Unfortunately there has been no reliable and simple method of identifying "high risk" patients within this group. The present report describes a number of novel and potentially important findings. Firstly, a chest radiograph, 12 lead ECG, and 24 hour ECG can provide information allowing the identification of patients at substantially increased risk of sudden death. Secondly, measurement of QT dispersion across leads VI-V6 provides useful prognostic information, whereas QT dispersion across the 12 lead ECG does not. Thirdly, in the present population of patients with mild to moderate CHF measurements of heart rate variability did not provide information identifying patients at risk of sudden death. In a previous report we showed that heart rate variability can predict progressive heart failure death. ${ }^{12}$ The potential mechanisms are discussed in detail in that report. Fourthly, QRS dispersion provides additional prognostic information to QRS maximum. Lastly, we have shown that the integration of four non-invasive measurements has the potential to identify patients at high risk of dying suddenly as opposed to dying non-suddenly over a five year period.

\section{Study population}

We studied patients with both ischaemic and non-ischaemic causes of CHF and we included patients with preserved systolic function. Our index performed well in identifying members of this heterogeneous group at risk of sudden death. Studies have suggested that the mechanisms of ventricular arrhythmia in ischaemic heart disease and nonischaemic cardiomyopathy may be different. Furthermore, more sophisticated investigation and treatments apply only to patients with ischaemic heart disease and impaired systolic function. When applied to this group our index performed even better, supporting its potential as an initial risk stratification tool for identifying patients with mild to 
Table 3 Characteristics of patients according to outcome

\begin{tabular}{|c|c|c|c|c|}
\hline Study population & Survivors & Sudden death & Non-sudden death & p Value \\
\hline UK-HEART $(n=553)$ & $\mathrm{n}=352$ & $n=67$ & $n=134$ & \\
\hline Age (years) & $61.8(9.6)$ & $62.4(9.9)$ & $64.2(9.2)$ & 0.002 \\
\hline Male sex & $74.4 \%$ & $73.1 \%$ & $82.8 \%$ & 0.121 \\
\hline NYHA class III & $29.8 \%$ & $49.3 \%$ & $57.5 \%$ & $<0.001$ \\
\hline LVEF (\%) & 44.0 (17) & $36.2(14)$ & $38.5(15)$ & $<0.001$ \\
\hline LVESD $(\mathrm{cm})$ & 4.88 (1.1) & $5.50(1.0)$ & $5.31(1.2)$ & $<0.001$ \\
\hline $\operatorname{LVEDD}(\mathrm{cm})$ & $6.08(0.9)$ & $6.53(0.9)$ & $6.41(1.1)$ & $<0.001$ \\
\hline CTR $(\%) \dagger$ & $0.51(0.48-0.55)$ & $0.57(0.52-0.60)$ & $0.55(0.50-0.60)$ & $<0.001$ \\
\hline NSVT present & $31.2 \%$ & $56.7 \%$ & $48.5 \%$ & $<0.001$ \\
\hline LVH present & $8.0 \%$ & $10.4 \%$ & $16.4 \%$ & 0.023 \\
\hline Heart rate (beats/min)† & $72.5(64-84)$ & $76.8(64-89)$ & $77.0(68-89)$ & 0.037 \\
\hline QRS dispersion $(\mathrm{ms}) \dagger$ & $41.3(34-49)$ & 47.0 (39-59) & $43.3(37-54)$ & 0.003 \\
\hline QTc dispersion leads V1-V6 (ms)† & $35.0(24-49)$ & $42.0(31-63)$ & $37.0(28-50)$ & 0.009 \\
\hline Sudden cardiac death score & $3.07(0.73)$ & $3.86(0.84)$ & $3.55(0.74)^{\star *}$ & $<0.001$ \\
\hline IHD and LVEF $<45 \%(n=262)$ & $n=148$ & $\mathrm{n}=38$ & $n=76$ & \\
\hline Age (years) & $61.7(8.2)$ & $63.7(8.5)$ & $64.0(8.8)$ & 0.106 \\
\hline Male sex & $81.1 \%$ & $73.7 \%$ & $89.5 \%$ & 0.091 \\
\hline NYHA class III & $40.5 \%$ & $60.5 \%$ & $56.6 \%$ & 0.019 \\
\hline LVEF (\%) & $30.9(8.5)$ & $28.2(10.1)$ & $29.9(8.5)$ & 0.224 \\
\hline $\operatorname{LVESD}(\mathrm{cm})$ & $5.46(0.9)$ & $5.78(1.0)$ & $5.78(1.0)$ & 0.027 \\
\hline $\operatorname{LVEDD}(\mathrm{cm})$ & $6.35(0.8)$ & $6.58(0.9)$ & $6.69(1.0)$ & 0.025 \\
\hline CTR $(\%) \dagger$ & $0.52(0.48-0.54)$ & $0.57(0.55-0.60)$ & $0.55(0.50-0.61)$ & $<0.001$ \\
\hline NSVT present & $34.5 \%$ & $65.8 \%$ & $50.0 \%$ & 0.001 \\
\hline LVH present & $4.7 \%$ & $13.2 \%$ & $13.2 \%$ & 0.051 \\
\hline Heart rate (beats/min)† & $74.6(66-83)$ & $76.9(60-89)$ & $75.2(66-89)$ & 0.573 \\
\hline QRS dispersion $(\mathrm{ms}) \dagger$ & $42.3(35-51)$ & $47.3(39-59)$ & $44.0(35-54)$ & 0.061 \\
\hline QTc dispersion leads V1-V6 (ms)† & $34.9(24-48)$ & $43.5(32-74)$ & $37.0(30-54)$ & 0.011 \\
\hline Sudden cardiac death score & $3.17(0.71)$ & $4.09(0.73)$ & $3.61(0.79)^{*}$ & $<0.001$ \\
\hline
\end{tabular}

moderate ischaemic heart failure at increased risk of sudden death, who may benefit from more intensive evaluation and treatment.

\section{Electrical inhomogeneity and its relation to sudden death}

It is likely that the major electrical feature necessary for the development of sustained ventricular tachyarrhythmias and thus sudden death is electrical inhomogeneity. ${ }^{21}$ In the normal heart there is some degree of electrical inhomogeneity due to the different cell types present. This inhomogeneity becomes more pronounced in the failing heart producing conditions that are favourable for the induction of sustained re-entrant ventricular tachycardia. As the QRS complex is thought to represent ventricular depolarisation, increased QRS dispersion on the 12 lead ECG may represent inhomogeneity of depolarisation, creating an environment that substantially increases the likelihood of initiating a re-entry circuit. A link between inhomogeneous electrical depolarisation and arrhythmic death in tetralogy of Fallot, ${ }^{22}$ arrhythmic right ventricular cardiomyopathy, ${ }^{23}$ and severe $\mathrm{CHF}^{24}$ has been postulated. The present study is the first to address this question in a large prospective study of patients with mild to moderate CHF.

The QT interval is thought to represent electrical repolarisation and it has been proposed that increased QT dispersion is indicative of inhomogeneous repolarisation. No study has shown unequivocally that measurements of QT dispersion obtained from a standard 12 lead ECG are related to increased risk of sudden death in CHF. ${ }^{11}{ }^{25}$ The present study confirms this but has produced the novel finding that the measurement of QT dispersion across leads V1-V6 is a potential prognostic tool to predict future arrhythmic events. This finding may be due to these measurements being more closely related to inhomogeneity of repolarisation across a scarred left ventricle when compared with QT dispersion measured from all 12 ECG leads. ${ }^{14}$ It is a finding that warrants further study.

\section{Cardiothoracic ratio and sudden death}

We showed that the greater a patient's cardiothoracic ratio, the greater their risk of sudden death. In our dataset, there was only a weak correlation between cardiothoracic ratio and left ventricular cavity internal dimensions. The cardiothoracic ratio may therefore provide information about overall cardiac morphology and interaction with the lungs. Myocardial stretch due to mechanical loading can increase refractoriness, trigger afterdepolarisations and ventricular extrasystoles, and slow conduction favouring re-entry and arrhythmia. Consistent with this hypothesis, a recent study from Berger and colleagues $^{26}$ showed that increased plasma B type natriuretic peptide concentrations (a marker of ventricular stretch) are an independent predictor of sudden death in patients with severe CHF (as shown by ejection fractions less than $20 \%$ ). These findings suggest that mechanoelectrical interaction may have an important role in the pathogenesis of sudden death in CHF. The positive predictive value of plasma B type natriuretic peptide concentrations to predict sudden death in this study, however, was less than $20 \%$. The integrated index derived from UK-HEART had a positive predictive value of about $40 \%$, a value that to our knowledge is the best of any current method of predicting sudden death in patients with CHF. ${ }^{4}$

Relevant to our finding of a relation between cardiothoracic ratio and sudden death is the finding that the occurrence of ventricular arrhythmia and sudden death has been shown to be closely related to lung function. ${ }^{27}{ }^{28}$ Substantial cardiomegaly in patients with CHF may interfere with 
normal lung function, providing a further mechanism to explain the observed relation between cardiomegaly and sudden cardiac death.

\section{Effect of non-sustained ventricular tachycardia on risk of sudden cardiac death}

The majority of studies exploring the relation between nonsustained ventricular tachyarrhythmias and sudden cardiac death have been retrospective analyses of patients with severe heart failure taking part in trials of therapeutic agents..$^{29}$ In a long term prospective study designed specifically to evaluate prognostic markers, we have confirmed that the presence of non-sustained ventricular tachyarrhythmia in patients with mild to moderate CHF is an ominous sign, increasing the risk of sudden death twofold. The relation between non-sustained ventricular tachycardia and sustained ventricular tachycardia is far from clear. ${ }^{21}$ Despite this, in our cohort non-sustained ventricular tachycardia in association with evidence of cardiac dilatation and inhomogeneity of depolarisation/repolarisation was a combination that clearly identified patients at increased risk of sudden (presumably) arrhythmic death.

\section{Performance of sudden cardiac death index}

The present analysis aimed at constructing an index that could identify patients specifically at increased risk of sudden death. The mechanisms underlying this are complex and multifactorial. In keeping with this the index required four independent predictors from a rigorous multivariate analysis. The C statistics of 0.71 for the whole population and 0.76 for patients with systolic dysfunction secondary to ischaemic heart disease illustrate the ability of our index to discriminate between patients at increased risk of sudden death per se.

An important role of an index predicting sudden death is its ability to discriminate between patients who die suddenly and those who die non-suddenly. When comparing the scores of these groups in our whole population and in patients with ischaemic heart disease underlying their CHF, our index allowed us to distinguish one group from the other. As with all prognostic indices the present data should be tested in different heart failure populations in different geographical locations to assess whether its discrimination degrades in separate cohorts. ${ }^{31}$ If the value of this index holds up in other studies, it offers a means of cost effectively targeting expensive interventions into a high risk subgroup of patients with CHF. Preventing sudden death is very important for patients with mild to moderate CHF, since their pump function is adequate to maintain a good quality of life for long term survivors.

\section{Study limitations}

As in all studies of this nature, classification of death is a potential problem, in particular attribution of sudden death to cardiac arrhythmia. We used strict predefined criteria for classification of death, which was carried out by senior physicians blinded to patient details. It has been suggested that sudden death in heart failure is not just due to arrhythmia and may be due to bradycardia or electromechanical dissociation in some patients. ${ }^{32}$ It is likely, however, that these are a feature of more severe heart failure, whereas our cohort had mild to moderate CHF based on both symptomatic and echocardiographic data. Another potential limitation of the present study is that while the majority of patients were stabilised with angiotensin converting enzyme inhibitors, the study commenced and follow up finished just as the landmark trials of $\beta$ blockers in mild to moderate CHF were being published. It would hence not be possible to have five year follow up of patients all taking these agents at present. However, in these studies patients with an ejection fraction
$>40 \%$ were excluded. The UK-HEART study was designed such that our population represented more accurately the usual CHF population with about $40 \%$ having preserved ejection fractions. There is thus no evidence as yet to support $\beta$ blocker use in a substantial proportion of the UK-HEART and wider CHF population. Despite this our findings should be tested in a population taking $\beta$ blockers. The present study excluded diabetic patients and, therefore, we cannot extrapolate from our data to the diabetic population.

\section{Conclusion}

The present study shows that it is possible to identify patients with mild to moderate CHF at increased risk of sudden death from 12 lead and 24 hour ECGs and a posteroanterior chest radiograph. Whether these new findings can be used in tailoring treatment or targeting investigations warrants further studies.

\section{ACKNOWLEDGEMENTS}

MTK, AMS and KAAF are supported by The British Heart Foundation.

\section{Authors' affiliations}

M T Kearney, A M Shah, N Gall, King's College, London, UK K A A Fox, A Flapan, Department of Cardiology, University of Edinburgh, Edinburgh, UK

A J Lee, R J Prescott, Medical Statistics Unit, University of Edinburgh W P Brooksby, P D Batin, Pontefract and Wakefield Hospitals, UK D L Eckberg, Medical College of Virginia at Virginia Commonwealth University, Virginia, USA

A G Zaman, Freeman Hospital, Newcastle upon Tyne, UK

H S Lindsay, Bradford Royal Infirmary, Bradford, UK

J Nolan, North Staffordshire Cardiac Centre, UK

\section{REFERENCES}

1 Gomberg-Maitland M, Baran DA, Fuster V. Treatment of congestive heart failure: guidelines for the primary care physician and the heart failure specialist. Arch Intern Med 2001; 161:342-52.

2 Anon. Effect of metoprolol CR/XL in chronic heart failure: metoprolol CR/XL randomised intervention trial in congestive heart failure (MERIT-HF). MERIT-HF study group. Lancet 1999;353:2001-7.

3 Kannel WB, Plehn JF, Cupples LA. Cardiac failure and sudden death in the Framingham study. Am Heart J 1988; 1 15:869-75.

4 Sweeney MO. Sudden death in heart failure associated with reduced left ventricular function: substrates, mechanisms, and evidence-based management, part I. J Pacing Clin Electrophysiol 2001;24:871-87.

5 Uretsky BF, Sheahan RG. Primary prevention of sudden cardiac death in heart failure: will the solution be shocking? J Am Coll Cardiol 1997;30:1589-97.

6 Moss AJ, Hall WJ, Cannom DS, et al. Improved survival with an implanted defibrillator in patients with coronary disease at high risk for ventricular arrhythmia. Multicentre automatic defibrillator implantation trial investigators. N Engl J Med 1996;335:1933-40

7 Moss AJ, Zareba W, Hall WJ, et al. Prophylactic implantation of a defibrillator in patients with myocardial infarction and reduced ejection fraction. N Engl J Med 2002;346:877-83.

8 De Vreede-Swagemakers JJ, Gorgels AP, Dubois-Arbouw WI, et al. Out of hospital cardiac arrest in the 1990's: a population based study in the Maastricht area on incidence, characteristics and survival. J Am Coll Cardiol 1997;30:1500-5.

9 Ezekowitz JA, Armstrong PW, McAllister FA. Implantable cardioverter defibrillators in primary and secondary prevention: a systematic review of randomised controlled trials. Ann Intern Med 2003;138:445-52.

10 Nolan J, Batin PD, Andrews R, et al. Prospective study of heart rate variability and mortality in chronic heart failure: results of the United Kingdom heart failure evaluation and assessment of risk trial (UK-HEART). Circulation 1998;98:1510-6.

11 Brooksby P, Batin PD, Nolan J, et al. The relationship between QT intervals and mortality in ambulant patients with chronic heart failure: the United Kingdom heart failure evaluation and assessment of risk trial (UK-HEART). Eur Heart J 1999;20:1335-41.

12 Kearney MT, Fox KA, Lee AJ, et al. Predicting death due to progressive heart failure in patients with mild-to-moderate chronic heart failure. J Am Coll Cardiol 2002;40:1801-8.

13 Senni M, Redfield MM. Heart failure with preserved systolic function: a different natural history? J Am Coll Cardiol 2001;38:1277-82.

14 Statters DJ, Malik M, Ward DE, et al. QT dispersion: problems of methodology and clinical significance. I Cardiovasc Electrophysiol 1994;5:672-85. 
15 Sokolow M, Lyon TP. The ventricular complex in left ventricular hypertrophy as obtained by unipolar precordial and limb leads. Am Heart $J$ 1949;37:161-86.

16 Saul JP, Arai Y, Berger RD, et al. Assessment of autonomic regulation in chronic congestive heart failure by spectral heart rate analysis. Am J Cardiol 1988;61:1292-9.

17 Hinkle LE, Thaler HT. Clinical classification of cardiac deaths. Circulation 1982;65:457-64.

18 Cox DR. Regression models and life tables. J R Stat Soc 1972;34:187-220.

19 Harrell FE Jr, Lee KL, Mark DB. Multivariable prognostic models: issues in developing models, evaluating assumptions and adequacy, and measuring and reducing errors. Stat Med 1996;15:361-87.

20 Hanley JA, McNeil BJ. The meaning and use of the area under a receiver operating characteristic (ROC) curve. Radiology 1982;143:29-36.

21 Babuty D, Lab MJ. Mechanoelectrical contributions to sudden cardiac death. Cardiovasc Res 2001;50:270-9.

22 Gatzoulis MA, Balaii S, Webber SA, et al. Risk factors for arrhythmia and sudden cardiac death late after repair of tetralogy of Fallot: a multicentre study. Lancet 2000;356:975-81.

23 Turrini P, Corrado DC, Basso C, et al. Dispersion of ventricular depolarisationrepolarisation a noninvasive marker for risk stratification in arrhythmogenic right ventricular cardiomyopathy. Circulation 2001;103:3075-80.

24 Anastasiou-Nana MI, Nanas JN, Karagounis LA, et al. Relation dispersion of QRS and QT in patients with advanced congestive heart failure to cardiac and sudden death mortality. Am J Cardiol 2000;85:1212-7.
25 Brendorp B, Elming $\mathrm{H}$, Jun $\mathrm{L}$, et al. QT dispersion has no prognostic information for patients with advanced congestive heart failure and reduced left ventricular systolic function. Circulation 2001;103:831-5.

26 Berger R, Huelsman M, Strecker K, et al. B-type natriuretic peptide predicts sudden death in patients with chronic heart failure. Circulation 2002; 105:2392-7.

27 Engstrom G, Wollmer P, Hedblad B, et al. Occurrence and prognostic significance of ventricular arrhythmia is related to pulmonary function: a study from "men born in 1914," Malmo, Sweden. Circulation 2001;103:3086-91.

28 Friedman GD, Klatsky AL, Siegelaub AB. Lung function and risk of myocardial infarction and sudden cardiac death. N Engl J Med 1976;294:1071-5.

29 Teerlink JR, Jalaluddin M, Anderson S, et al. Ambulatory ventricular arrhythmias in patients with heart failure do not specifically predict an increased risk of sudden death. Promise investigators. Circulation 2000;101:40-6.

30 Doval HC, Nul DR, Grancelli HO, et al. Non-sustained ventricular tachycardia in severe heart failure: independent marker of increased mortality due to sudden death: GESICA-GEMA investigators. Circulation 1996;94:3198-203.

31 Pogwizd SM, McKenzie JP, Cain ME. Mechanisms underlying spontaneous and induced ventricular arrhythmias in patients with idiopathic dilated cardiomyopathy. Circulation 1998:98:2404-14.

32 Luu M, Stevenson W, Stevenson L, et al. Diverse mechanisms of unexpected cardiac arrest in advanced heart failure. Circulation 1989;80:1675-80.

\section{IMAGES IN CARDIOLOGY}

\section{Echolucent neointimal hyperplasia "dark wall" after sirolimus eluting stent implantation}

\begin{abstract}
A 45 year old man was admitted because of exertional angina. Coronary angiography revealed a $70 \%$ stenosis in the left circumflex artery (LCX). He was enrolled in the US SIRIUS study to evaluate the efficacy of sirolimus eluting stents in de novo native coronary lesions. A dramatic reduction of restenosis has previously been demonstrated using sirolimus eluting stents. A $3.0 \times 18 \mathrm{~mm}$ sirolimus eluting stent (Cordis, Johnson \& Johnson, Miami, Florida, USA) was deployed in the distal LCx lesion. Eight months later, angiography and intravascular ultrasound (IVUS) were performed according to the study protocol (see fig), although he had been asymptomatic. IVUS imaging demonstrated nonobstructive, eccentric echolucent tissue in the proximal part of the stent, which corresponded to the angiographic mild, eccentric luminal narrowing. No further procedure was performed.

Echolucent tissue, which is termed "black hole" and more appropriately "black wall", has been reported after intracoronary brachytherapy. The tissue specimens obtained by atherectomy in a limited number of cases demonstrated that the echolucent findings appeared to be caused by a hypocellular matrix with areas of proteoglycan. Proteoglycan has a high water content that may explain the IVUS features. While we do not have histological data on this patient, the echolucent tissue in this patient may likewise be caused by hypocellular neointimal hyperplasia rich in proteoglycan.
\end{abstract}
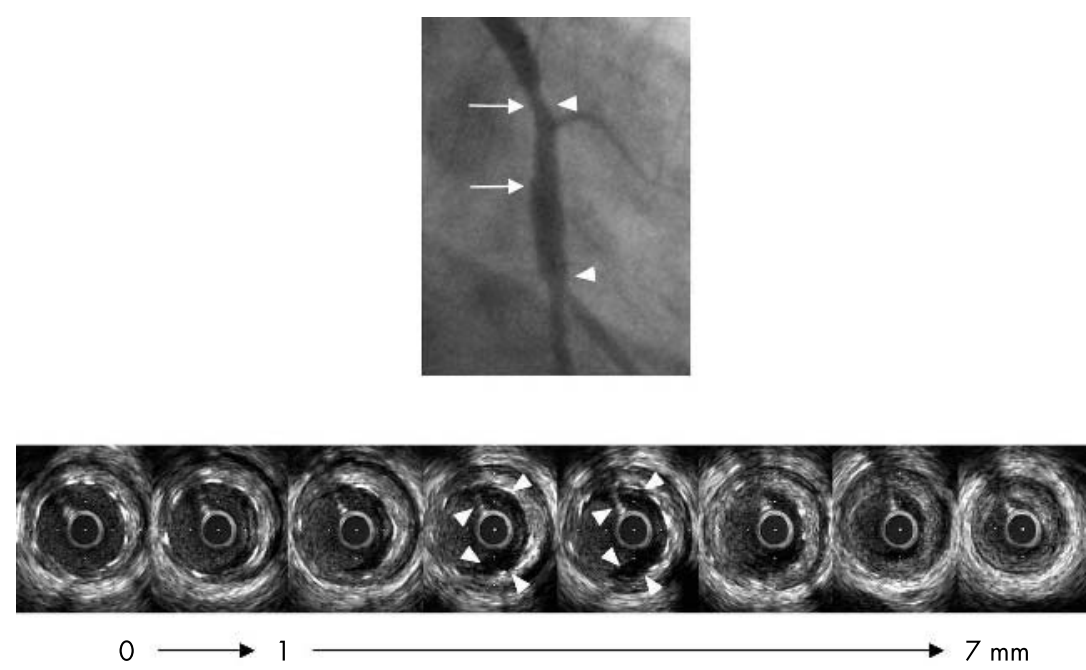

Distal

Proxima

White arrows in angiogram (top) correspond to the segment of the circumflex coronary artery depicted in the intravascular ultrasound (IVUS) imaging sequence (bottom). Arrowheads in angiogram indicate the edges of the stent. IVUS images are displayed from distal (on left) to proximal (on right). Individual images are $1 \mathrm{~mm}$ apart to illustrate a $7 \mathrm{~mm}$ length of the proximal part of the stent. Each image except the most right and left demonstrates crescent shaped, echolucent neointima (white arrowheads) inside the stent.
M Hirose

Y Kobayashi

$M$ B Leon

mleon@crf.org 